\title{
Eine Methode zur colorimetrischen Bestimmung von Ammoniak in Meerwasser
}

\author{
R. Benesch P. MANgelsdorf \\ Biologische Anstalt Helgoland (Meeresstation); \\ Helgoland, Bundesrepublik Deutschland
}

\begin{abstract}
A method for colorimetric determination of ammonia in sea water. A method is described which measures the amount of an ammonium compound colorimetrically, instead of measuring the consumption of oxidizing matter. The method is based on the formation of a blue quinoid dye, with sodium salicylate as phenolic, and sodium derivative of dichlorcyanuric acid as halogenic reagent. Most non-ammonium compounds occuring naturally in sea water do not interfere with this reaction. The method is applicable within a wide salinity range manually as well as automatically. Manual determination requires strict linear interdependence between concentration and extinction. In the automatical determination, concentration is expressed by the peak level; this independence from linearity facilitates more exact measurements at lower ammonia concentrations. For shipboard investigations the preparation of reagents is simplified to eleminate handling errors. Exact control of temperature and reaction time is essential. The reproducibility of the method is approximately $3 \%$ within the range investigated: 0.35 to $16.60 \mu \mathrm{g}$-at $\mathrm{NH}_{4}^{+}-\mathrm{N} / \mathrm{l}$.
\end{abstract}

\section{EINLEITUNG}

Seit 1961 wird an der Biologischen Anstalt Helgoland im Rahmen hydrographisch-chemischer Untersuchungen der Ammoniakgehalt des Meerwassers nach der Methode von GILlBRicht (1961) bestimmt. Dabei wird oxidierbare Substanz in alkalischer Lösung mit Natriumhy pobromit ( $\mathrm{NaOBr}$ ) zerstört und das überschüssige $\mathrm{NaOBr}$ in saurer Lösung mit Bordeauxrot reduziert. Der Verbrauch an Bordeauxrot wird im ZEIss-Photometer Elko II gemessen. Durch die besondere Methodik werden außer Ammoniak die meisten der anwesenden Substanzen nicht angegriffen. Lediglich einige leicht oxidierbare Stoffe (z. B. Fe [II], Teile der gelösten organischen Substanz, die Aminogruppen einiger Aminosäuren und Amine) werden mit erfaßt. Der Oxidationsmittelverbrauch ist also stark von der qualitativen Zusammensetzung der jeweiligen Wasserprobe abhängig. Insbesondere wirken sich reduzierende Medien und Unterschiede im Planktongehalt auf den Oxidationsmittelverbrauch aus. Die Methodik der $\mathrm{NaOBr}$-Bordeauxrot-Methode bringt einen weiteren Nachteil mit sich: $\mathrm{Da}$ hierbei zwei veränderliche Lösungen gegeneinander photometriert werden müssen, läßt sich das Verfahren nur schlecht mit dem Autoanalyzer ausnutzen. 
Es wurde daher versucht, die Messung des Oxidationsmittelverbrauches durch eine direkte chemische Reaktion des Ammoniaks zu ersetzen. Die in dieser Richtung bisher entwickelten Bestimmungsmethoden beruhen meistens auf der BERTHELOTschen Reakrion (1859), der Bildung eines Indophenolblaues aus Phenol mit Hypochlorit als Halogenträger in Gegenwart von Ammoniak (SAGr 1966, Solorzano 1969, KorolefF 1970, HEAD 1971). NEWELI (1967) benutzt Chloramin $T$ als Halogenträger und extrahiert das entstandene Indophenol mit n-Hexanol. Während die letzte Methode insbesondere während des Bordbetriebes zu umständlich ist, besteht bei der Reaktion des Ammoniaks mit Hypochlorit im alkalischen Medium die Möglichkeit einer Oxidation zum Nitrit-Ion, eine Reaktion, die von Richards \& KLETSCH (1964) direkt zum Nadhweis von Ammoniak ausgenutzt wird.

Bei der hier beschriebenen Methode wird dagegen Natrium-Dichlorcyanurat [Dichlor-s-triazin-2,4,6 $(1 \mathrm{H}, 3 \mathrm{H}, 5 \mathrm{H})$-trion Natriumderivat] als Halogenträger benutzt. Das Dichlorcyanurat bringt gegenüber Hypochlorit den Vorteil einer größeren Beständigkeit der Reaktionslösung und gegenüber Chloramin T die Möglichkeit einer einfacheren Durchführung der Reaktion. Dabei wurden die Arbeiten von ReardoN et al. (1966) und WAGNER (1970) zugrunde gelegt. Sie bestimmten das Ammoniak im Blut bzw. im Süßwasser mit Natrium-Dichlorcyanurat als Halogenträger. Hierbei wird nur eine veränderliche Lösung gegen eine konstante Gegenlösung photometriert, so daß das Verfahren auch für den Einsatz im Autoanalyzer geeignet ist.

Um die Vorteile der sofortigen Messung des Ammoniakgehaltes im Meerwasser auf Forschungsfahrten mit dem Autoanalyzer ausnutzen zu können, wurde die Dichlorcyanurat-Methode an die Gegebenheiten des Technixon Autoanalyzers angepaßt. Diese Methode wurde auch deshalb gewählt, weil die einzelnen Reagenzien schon vorbereitet an Bord genommen werden können. Dadurch sollen die unter den erschwerten Bordbedingungen etwa durch Pipettieren oder Einfüllen in die Meßkiivetten hervorgerufenen Fehler bei manuellen Arbeiten möglichst wegfallen.

\section{CHEMISMUS}

Voraussetzung einer Bestimmung des Ammoniaks im Meerwasser ist, daß die Reaktion im alkalischen Medium abläuft, da sonst das Ammoniak als Ammonium-Ion gebunden vorliegt und als solches nicht in die Reaktion eingeht.

Zur Bestimmung des Ammoniaks im Meerwasser wurde aus den genannten Gründen die Bildung einer chinoiden Verbindung gewählt. Um die Entstehung des Farbstoffes zu beschleunigen, muß entweder bei höheren Temperaturen oder in Gegenwart eines Katalysators gearbeitet werden. Als Katalysatoren eignen sich Ionen, die in mindestens zwei Wertigkeiten vorkommen (Fe-, Cu- oder Mn-Ion). Luвochinsкy \& ZaLTA (1954) schlugen als Katalysator das Nitroprussid-Natrium vor, das besonders günstig ist, da es die Reaktion stark beschleunigt und keine zu kräftige Eigenfärbung besitzt. Im Nitroprussid-Ion liegen das $\mathrm{Fe}+2$ wertig und das $\mathrm{NO}+1$ wertig vor. Die katalytische Wirkung kann möglicherweise auch auf einem Austausch der in alkalischer Lösung vorliegenden Nitritgruppe gegen Ammoniak beruhen: 


$$
\begin{aligned}
& \mathrm{Na}_{2}\left[\mathrm{Fe}(\mathrm{CN})_{5} \mathrm{NO}\right] \stackrel{+2 \mathrm{NaOH} \backslash}{\rightleftharpoons} \mathrm{Na}_{4}\left[\mathrm{Fe}(\mathrm{CN})_{5} \mathrm{NO}_{2}\right]+\mathrm{H}_{2} \mathrm{O} \\
& \mathrm{Na}_{4}\left[\mathrm{Fe}(\mathrm{CN})_{5} \mathrm{NO}_{2}\right] \stackrel{\mathrm{NH}_{3} \backslash}{\rightleftharpoons} \mathrm{Na}_{3}\left[\mathrm{Fe}(\mathrm{CN})_{5} \mathrm{NH}_{3}\right]+\mathrm{NaNO}_{2}
\end{aligned}
$$

Die Art der Reaktion, die zu der Farbstoffbildung führt, ist noch weitgehend ungeklärt. Wahrscheinlich bildet sich intermediär ein Chinon-Chlorimid (KorolefF 1970).

Um die Ausfällung der Erdalkalien in alkalischer Lösung zu verhindern, muß ein Chelatorsystem benutzt werden, das die Erdalkalien gut in Lösung hält, die Reaktion aber nicht stört. Graham-Salz $\left(\mathrm{NaPO}_{3}\right)_{n}$ reicht für Meerwasser mit seinem relativ hohen Erdalkaligehalt nicht aus. Versuchsreihen mit Athylendiammintetraessigsäure (ADTE) zeigten, daß bei geringer Konzentration die Erdalkalien zum Teil noch ausgefällt werden, bei höherer Konzentration an ADTE die Farbbildung jedoch ganz erheblich verzögert wird. Bei Einsatz von größeren Konzentrationen muß man die Reaktionstemperatur stark erhöhen und gelangt trotzdem nur zu schlechten Farbausbeuten. Daraus könnte man schließen, daß vielleicht das als Katalysator eingesetzte Nitroprussid-Ion durch das ADTE ebenfalls eingefangen wird. Daher wurde nach Solorzano (1969) und KorolefF (1970) ein NaOH-Natriumcitrat-Puffer benutzt, der die Erdalkalien gut in Lösung hält, die Reaktionsgeschwindigkeit aber nicht merklich beeinflußt.

\section{REAGENZIEN UND METHODE}

Für die Analyse werden folgende Reagenzien benötigt:

Reagenz 1: $12 \mathrm{~g}$ Natriumhydroxid (15 g Natriumhydroxid)* $+150 \mathrm{~g}$ Natriumcitrat in 11 destilliertem Wasser;

Reagenz 2: $170 \mathrm{~g}$ Natriumsalicylat $+1,2 \mathrm{~g}$ Nitroprussid-Natrium in 11 destilliertem Wasser;

Reagenz 3: 2 g Natrium-Dichlorcyanurat in 11 destilliertem Wasser;

Sperfflüssigkeit: Entsprechend dem zu erwartenden Salzgehalt der zu untersuchenden Lösung $(30-36 \%$ \%

Die mit * bezeichneten Angaben beziehen sich auf die anderen Konzentrationen, die bei der Untersuchung mit dem. Autoanalyzer zur Anwendung kommen. Die Lösung 1 ist beständig. Der pH-Wert von $12,94 \mathrm{bzw} .13,72^{*}$ sollte auf $\pm 0,2 \mathrm{pH}$ genau eingehalten werden. Reagenz 2 ist bei Zimmertemperatur ca. eine Woche beständig. Die Aufbewahrung erfolgt zweckmäßig im Dunklen oder in braunen Flaschen. Die Lösung dunkelt während des Stehens etwas nach, ohne jedoch ihre Reaktivität zu verändern. Lösung 3 sollte täglich frisch angesetzt werden. Für den Bordbetrieb empfiehlt es sich, das Natrium-Dichlorcyanurat in Tagesportionen einzeln in Glasgefäße einzuwiegen, oder aber in Mowiol-Folien einzuschmelzen (Polyvinylalkohol, Farbwerke HoEchst AG), die zusammen mit dem Reagenz in der entsprechenden Wassermenge gelöst werden können.

Bei der manuellen Bestimmung werden $50 \mathrm{ml}$ Meerwasser mit je $5 \mathrm{ml}$ der Reagenzien 1 und 2 versetzt und geschüttelt. Anschließend werden $5 \mathrm{ml}$ der Lösung $3 \mathrm{zu-}$ geführt. Die Proben bleiben eine Stunde bei Zimmertemperatur oder $20 \mathrm{~min}$ bei $37^{\circ} \mathrm{C}$ 
im Dunklen stehen. Nach Abkühlung auf Zimmertemperatur $(15 \mathrm{~min})$ werden die Proben mit $35 \mathrm{ml}$ destilliertem Wasser aufgefüllt. Im ZeIss-Photometer Elko II wird mit Filter I 66 in der 1-cm-Küvette sofort die Durchlässigkeit gemessen und daraus die Extinktion $\left(\mathrm{E} \times 10^{4}\right)$ berechnet. Das Absorptionsmaximum liegt bei $670 \mathrm{~nm}$. Als Gegenlösung dient sterilfiltriertes Meerwasser (Millipore-Membranfilter $0,45 \mu \mathrm{m}$ ).

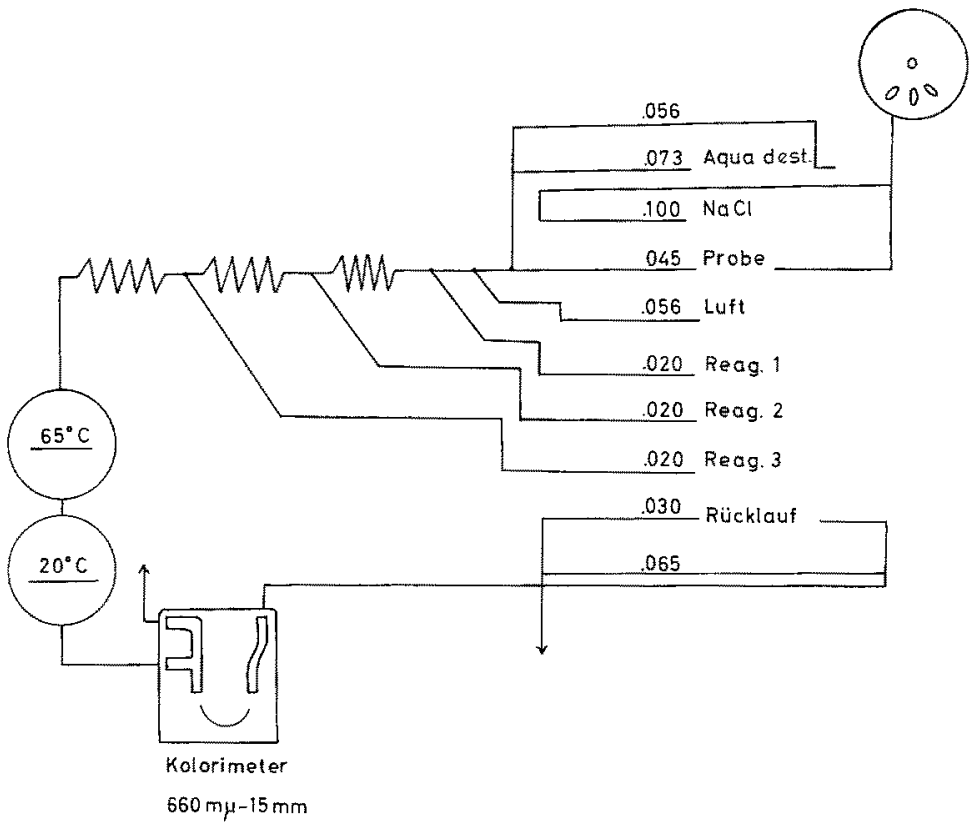

Abb. 1: Fließdiagramm des Tednikon Autoanalyzers zur Bestimmung der Ammoniak-Konzentration nach der Dichlorcyanurat-Methode. Reaktionszeit 2,5 min bei $65^{\circ} \mathrm{C}$. Filter $660 \mathrm{~nm}$, Küvette $15 \mathrm{~mm}$

Wichtig ist, daß die äußeren Bedingungen genau eingehalten werden. Besonders beachtet werden sollte die Einhaltung einer bei allen Messungen konstanten Reaktionstemperatur und -zeit sowie die Ausschaltung des Lichteinflusses auf die Reagenzien und die Reaktion. Nichteinhaltung dieser Bedingungen führt zu einer Anderung des Anstiegswinkels der Ammoniak-Eichkurve, da diese Faktoren die Einstellung des Gleichgewichtes der Farbstoffbildung beeinflussen.

Die Versuche mit dem Technikon Autoanalyzer wurden nach dem in Abbildung 1 skizzierten Fließdiagramm durchgeführt. Dabei wurde das Reaktionssystem soweit als möglich verkürzt, damit die Peaks und die Null-Linien nicht durch die relativ kurzen Reaktions- und Spülzeiten verzerrt werden. Die Reaktions- und Spülzeiten wurden aus dem gleichen Grunde weitestmöglich verlängert. Das Verhältnis Probenahme zu Spülzeit ist bei 10 Proben in der Stunde $1: 1$. Nur so konnte erreicht werden, daß die Peaks gut ausgebildet und die Basislinien (Reagenzienblindwert) zwischen den Peaks wieder erreicht werden. Eine auftretende Drift der Basislinie kann bei der Auswertung mit einem Lineal ausgeglichen werden. Bei längerer Versuchsdauer können Verzerrun- 
gen der Peaks und der Basislinie auftreten. Sie waren, wenn die Kurve durch einige zwischengeschaltete Probengefäße Sperrflüsigkeit nicht wieder auf die Normallinie gebracht werden konnte, auf Verunreinigungen durch Reagenz- bzw. Farbstoffablagerung im System zurückzuführen. Zur Reinigung wurde $\mathrm{HCl}(1: 4)$, Edisonite (Firma Merz \& Co.) und destilliertes Wasser benutzt.

Um die äußeren Bedingungen konstant zu halten, blieben die Reagenzien und das Meerwasser $2,5 \mathrm{~min}$ bei $65^{\circ} \mathrm{C}$ in einem Wasserbad und wurden anschließend in einer Kühlschlange auf $20^{\circ} \mathrm{C}$ abgekühlt. Die Temperaturkonstanz des Wasserbades betrug $\pm 0,1^{\circ} \mathrm{C}$.

\section{ERGEBNISSE UND DISKUSSION}

Um den Anwendungsbereich der Methode abzugrenzen, wurden bei der manuellen und der automatischen Bestimmung verschiedene Parameter variiert.

Die Farbstoffbildung ist sehr stark vom pH-Wert abhängig. Das Reaktionsgleichgewicht wird mit steigendem pH-Wert zu intensiverer Farbstoffbildung hin verschoben.

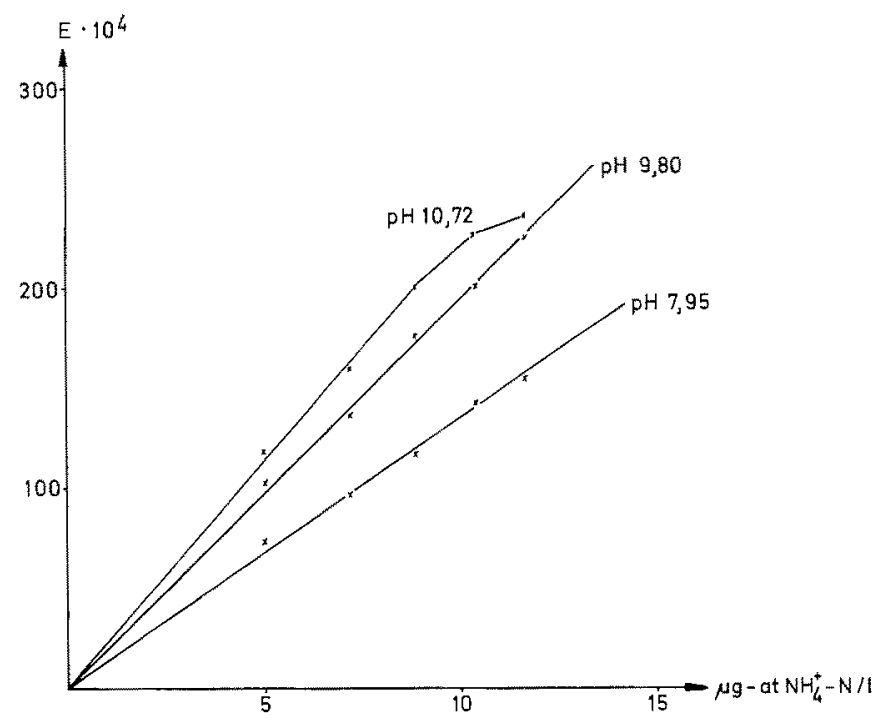

Abb. 2: Einfluß verschiedener $\mathrm{pH}$-Werte auf den Anstieg der Ammoniak-Eichkurve. Bei Messungen mit dem Elko II im Bereich zwischen pH 7,95 und 10,72 (Meerwasser + Reagenzien), der in Stufen von ca. 0,5 pH geändert wurde, erwies sich der $\mathrm{pH}$ 9,80 als am günstigsten. Eingezeichnet sind nur die Grenzwertkurven

Es wurden Meßreihen angesetzt, bei denen der pH-Wert zwischen 7,95 und 10,72 (Meerwasser + Reagenzien) in Stufen von ca. 0,5 pH geändert wurde. Die manuelle Messung zeigte, daß bei zu hohen $\mathrm{pH}$-Werten die Ammoniak-Eichkurve nicht mehr linear ist. Der günstigste Wert liegt bei pH 9,80 (Abb. 2). 
Bei der Bestimmung mit dem Autoanalyzer ist eine lineare Abhängigkeit zwischen Extinktion und Konzentration nicht erforderlich. Die Ammoniakkonzentration kann direkt durch die Peakhöhen ausgedrückt werden. Daher wurde der pH-Wert gegenüber der manuellen Methode erhöht, um eine größere Genauigkeit im Bereich geringerer Ammoniak-Konzentrationen zu erhalten. $\mathrm{Da}$ die Beziehung zwischen Peakhöhe und Konzentration nicht linear ist, kann nicht mit einem festen Faktor gerechnet werden. Aus diesem Grunde wurde ein Ableselineal entwickelt, das es gestattet, aus der Peakhöhe direkt die Konzentration an Ammoniak im Meerwasser abzulesen.

Die Reaktionen wurden außerdem in Kochsalzlösungen verschiedener Konzentrationen durchgeführt, um die Abhängigkeit vom Salzgehalt zu untersuchen. Testkonzentrationen waren: $20,08 \%, 25,16 \%, 29,22 \%, 31,38 \%, 33,02 \%, 37,26 \%$ und 42,88\%. Ein Einfluß konnte für den interessierenden Bereich (ca. 25-37\% nicht festgestellt werden. Erst oberhalb von $40 \%$ ändert sich der Anstiegswinkel der Ammoniak-Eichkurve, es treten dann auch Verzerrungen in der Peakform und thöhe auf. Diese weitgehende Unabhängigkeit vom Salzgehalt triff nicht nur für $\mathrm{NaCl}$ Lösungen, sondern auch für Meerwasser $\mathrm{zu}$, da die Erdalkalien durch das $\mathrm{NaOH}$ Natriumcitrat-Chelatorsystem in Lösung gehalten werden, den Gehalt an $\mathrm{OH}^{-}$-Ionen demnach nicht verändern können (MARTELL \& CALVIN 1958).

Bei der Zugabe von Ammonium-Ionen zu sterilfiltriertem Meerwasser erhält man mit der manuellen Methode die gleiche Steigung der Eichkurve wie bei der Zugabe zu $\mathrm{NaCl}$ Lösungen. Durch Extrapolation (Gehalt des Meerwassers an Ammoniak) kann die Gerade durch den Nullpunkt geführt werden. Bei der automatischen Bestimmung kann mit Hilfe des Ableselineals der Gehalt an Ammoniak aus dem Meerwasser zusätzlich zur zugegebenen Menge auf $\pm 3 \%$ genau gemessen werden.

Weiterhin wurde die Abhängigkeit von der Temperatur untersucht. Eine Erhöhung der Temperatur führt zu intensiverer Farbstoffbildung. Es handelt sich dabei offensichtlich um die Verschiebung eines temperaturabhängigen Reaktionsgleichgewichtes. Daher sollten die für die Eichungen gewählten Reaktionszeiten und -temperaturen sehr genau eingehalten werden. Bei der Bestimmung mit dem Autoanalyzer stellt die Reaktionszeit und -temperatur von $2,5 \mathrm{~min}$ und $65^{\circ} \mathrm{C}$ einen Kompromiß zwischen gewünschter hoher Temperatur und methodischer Zweckmäßigkeit an Bord dar.

Außerdem wurde die auch bei der manuellen Bestimmung notwendige Verdünnung des Meerwassers untersucht. Bei unverdünnten Proben ging die Verzerrung der Kurven aus dem Autoanalyzer so weit, daß anstelle der zu erwartenden Peaks sogar scheinbar negative Werte auftraten. Eine Verdünnungsreihe Meerwasser zu destilliertem Wasser von $1: 1,1: 2,1: 3,1: 4$ und $1: 5$ zeigte, daß - beginnend vom Verhältnis $1: 3$-die Peaks sauber ausgebildet waren.

Die Werte lassen sich auf ca. $\pm 3 \%$ genau reproduzieren. Dazu wurden bei der manuellen und bei der automatischen Bestimmung je 10 sich entsprechende Eichkurven auf einem Leuchttisch übereinandergelegt. Bei der manuellen Methode wurde der Anstiegswinkel der Eichkurven bestimmt und bei den mit dem Autoanalyzer gefundenen Werten der Peakhöhenunterschied gemessen. Die Streuung liegt bei beiden Methoden ca. 2-3\% um den eingegebenen Wert. Dabei wurden bei der manuellen Methode jeweils 10 Meßwerte für die verschiedenen Konzentrationen bestimmt. Die Messung mit 
dem Autoanalyzer erfolgte, indem 11 Probengefäße mit Lösung der gleichen Konzentration an Ammonium-Ionen gefüllt wurden. Der erste Peak wurde, da er bei allen Messungen weit außerhalb der Normalen lag, nicht ausgewertet. Es müssen also bei der

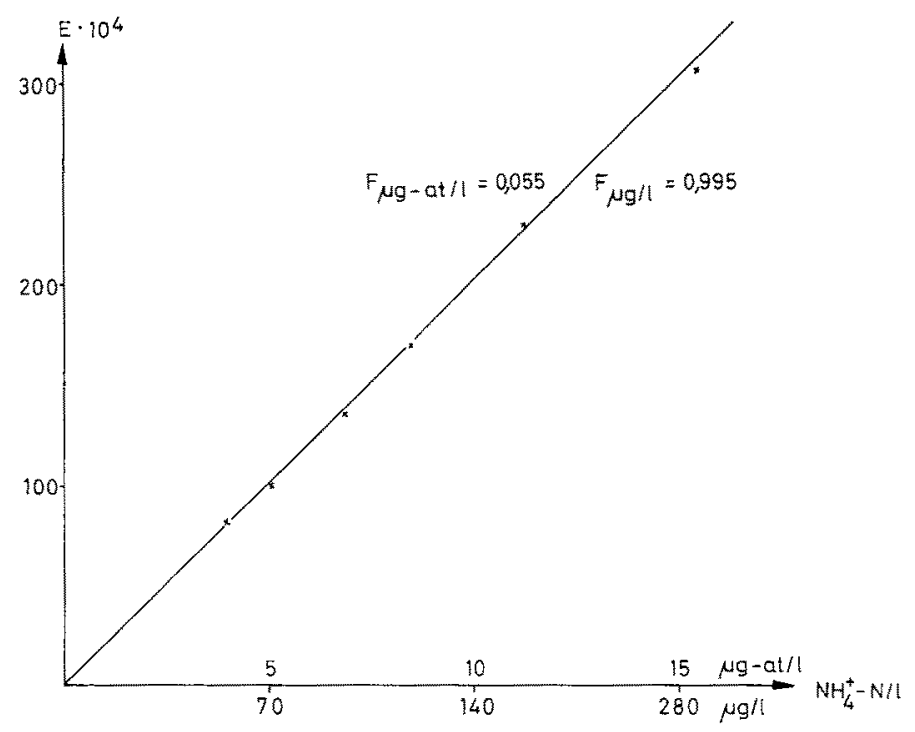

Abb. 3: Fichkurve für die manuelle Bestimmung von Ammoniak in sterilfiltriertem Meerwasser. Mittelwerte aus je 10 Meßdaten pro Meßpunkt. Im Elko II mit Filter I 66 in der 1 -cm-Küvette gemessen. Berechnet $\mathrm{F}_{\mu \mathrm{g}-\mathrm{at} / \mathrm{l}}=0,055$ und $\mathrm{F}_{\mu \mathrm{g} / \mathrm{l}}=0,995$. Gegenlösung ist sterilfiltriertes Meerwasser

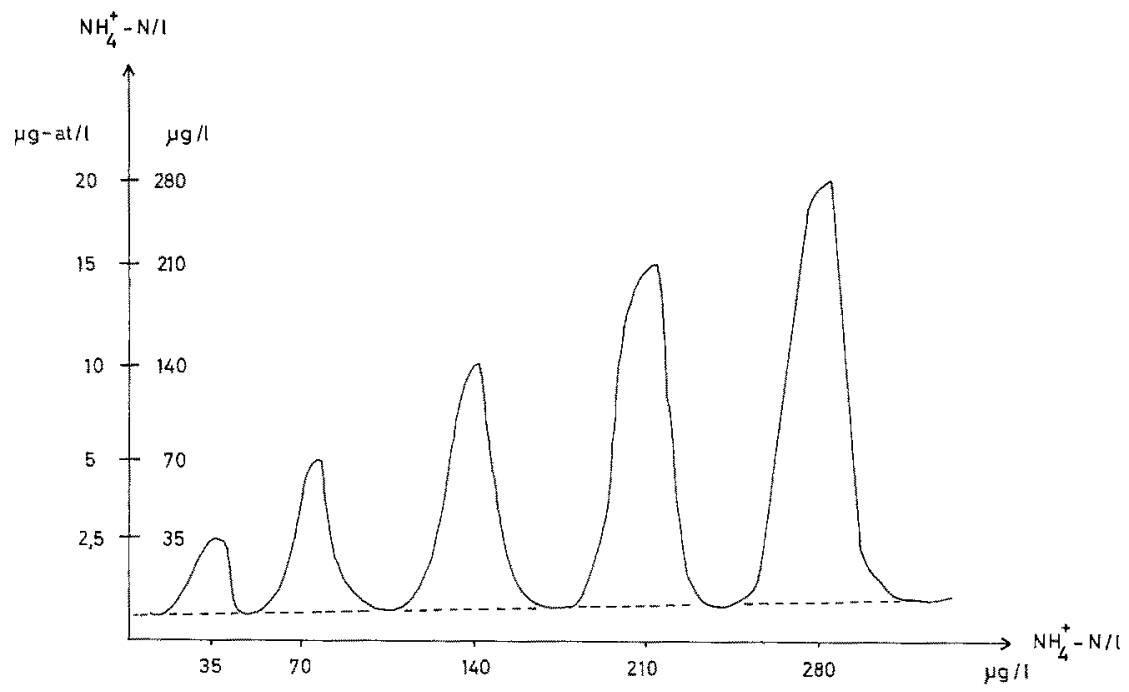

Abb. 4: Eichkurve für die Bestimmung von Ammoniak im Technikon Autoanalyzer. Da die Abhängigkeit von Konzentration und Extinktion nicht linear ist, wurden die Peakhöhen als Konzentration aufgetragen 
automatischen Bestimmung mindestens zwei Probengefäße mit der gleichen Lösung gefüllt und untersucht werden. Die Verzerrung des ersten Peaks ist wahrscheinlich auf Schlierenbildung, hervorgerufen durch Konzentrationsänderungen im System, zurückzuführen. Eine Verlängerung der Spïlzeit würde Abhilfe schaffen, wäre jedoch, da sie auf Kosten der Probenahmezeit ginge, nicht zweckmäßig.

Aus den verschiedenen Meßreihen ergaben sich die in Abbildung 3 für die manuelle und in Abbildung 4 für die automatische Bestimmung gegebenen Eichkurven. Die Meßbereiche, die mit der Dichlorcyanurat-Methode erfaßt werden, liegen bei der manuellen Methode zwischen ca. 1,65 und 16,65 $\mu$ g-at $\mathrm{NH}_{4}^{+}-\mathrm{N} / 1$, bei der Messung mit dem Technikon Autoanalyzer zwischen 0,35 und $16,60 \mu \mathrm{g}$-at $\mathrm{NH}_{4}^{+}-\mathrm{N} / \mathrm{l}$.

Um die mögliche Störung durch zugesetzte Aminosäuren zu untersuchen, wurden Vergleichsmessungen der NaOBr-Bordeauxrot-Methode und der DichlorcyanuratMethode durchgeführt. Es wurden $100 \mu \mathrm{g}$ Aminosäure zu $100 \mathrm{ml} \mathrm{NaCl-Lösung} \mathrm{(30 \% )}$ zugesetzt. Aus Tabelle 1 kann man entnehmen, daß ein Teil der Aminosäuren unter

Tabelle 1

Einfluß von zugesetzten Aminosäuren (jeweils $100 \mu \mathrm{g} / 100 \mathrm{ml} \mathrm{NaCl}$-Lösung $30 \%$ ) auf die Ammoniakbestimmung. Vergleich der $\mathrm{NaOBr}$-Bordeauxrot-Methode mit der DichlorcyanuratBestimmung. $+=100 \%$ des Aminosäurestickstoffes als Ammoniak bestimmt; $O=$ Aminogruppen sind teilweise erfaßt; $-=$ kein Aminosäurestickstoff gefunden

\begin{tabular}{|lcc|}
\hline Aminosäure & $\begin{array}{c}\text { NaOBr-Bordeauxrot- } \\
\text { Methode }\end{array}$ & $\begin{array}{c}\text { Dichlorcyanurat- } \\
\text { Methode }\end{array}$ \\
\hline Alanin & 0 & + \\
Cystein & + & \pm \\
Cytosin & + & \pm \\
Glycin & + & \pm \\
Lysin & + & - \\
Methionin & - & - \\
\hline
\end{tabular}

den Versuchsbedingungen durch das $\mathrm{NaOB}$ leichter angegriffen wird, als durch das Natrium-Dichlorcyanurat. Nach den Befunden von BoHLing (1970) für April/Mai 1968 entspricht die Menge an freien gelösten Aminosäuren in der Nordsee vor Helgoland, bezogen auf den Stickstoff, einer Ammoniakmenge von ca. 0,33 bis $0,89 \mu g$-at $\mathrm{NH}_{4}^{+}-\mathrm{N} / \mathrm{l}$.

Um die Ergebnisse der Ammoniak-Bestimmung nach dem Dichlorcyanurat-Verfahren abzusichern, wurden die manuelle und die automatische Bestimmung mit dem NaOBr-Bordeauxrot-Verfahren verglichen (Tab. 2). Das sterilfiltrierte Meerwasser hatte einen Gehalt von 4,16 $\mu$ g-at $\mathrm{NH}_{4}^{+}-\mathrm{N} / 1$. Der Salzgehalt betrug 30,12\%. Die mit * bezeichneten Proben wurden in NaCl-Lösung (30\%) angesetzt. Dabei zeigte sich, $\mathrm{da} \beta$ bei geringen Ammoniak-Konzentrationen (unter $1,9 \mu \mathrm{g}$-at $\mathrm{NH}_{4}^{+}-\mathrm{N} / \mathrm{l}$ ) die Bestimmung mit dem Autoanalyzer am genauesten ist. Bei Werten von mehr als 11,6 $\mu$ g-at $\mathrm{NH}_{4}^{+}-\mathrm{N} / 1$ gibt die NaOBr-Bordeauxrot-Methode die genauesten Ergebnisse.

Ferner wurden Proben von Terminbeobachtungen von der Station HelgolandReede nach den beiden Methoden vergleichsweise untersucht. Aus Tabelle 3 ist zu er- 
Tabelle 2

Vergleich der Bestimmung des Ammoniak-Gehaltes in Kochsalzlösung (30\%0, mit * bezeichnete Werte) und in Meerwasser mit der NaOBr-Bordeauxrot-Methode und der Dichlorcyanurat-Methode. Das Meerwasser hatte einen Gehalt von 4,16 $\mu \mathrm{g}-$ at $\mathrm{NH}_{4}^{+}-\mathrm{N} / \mathrm{l}$, der Salzgehalt betrug $30,12 \%$

\begin{tabular}{|c|c|c|c|}
\hline \multirow[t]{2}{*}{$\begin{array}{l}\text { Zugabe in } \\
\mu \text {-at } \mathrm{NH}_{4}^{+}-\mathrm{N} / 1\end{array}$} & \multirow{2}{*}{$\begin{array}{l}\text { Gefunden mit der } \\
\mathrm{NaOBr}-\mathrm{Bordeaux} \\
\text { rot-Methode in } \\
/ 4 \mathrm{~g} \text {-at } \mathrm{NH}_{4}^{+}-\mathrm{N} / 1\end{array}$} & \multicolumn{2}{|c|}{$\begin{array}{l}\text { Gefunden mit der Dichlorcyanurat- } \\
\text { Methode in } \mu \mathrm{g} \text {-at } \mathrm{NH}_{4}^{+}-\mathrm{N} / 1\end{array}$} \\
\hline & & manuell & Autoanalyzer \\
\hline $0,39 *$ & 0,46 & 0,53 & 0,39 \\
\hline $0,78^{*}$ & 0,83 & 0,95 & 0,78 \\
\hline $1,55^{*}$ & 1,86 & 1,65 & 1,56 \\
\hline $1,94^{*}$ & 1,96 & 1,99 & 1,95 \\
\hline $3,88^{*}$ & 3,89 & 3,89 & 3,88 \\
\hline 0,39 & 4,63 & 4,55 & 4,52 \\
\hline 0,78 & 4,98 & 4,92 & 4,91 \\
\hline 1,55 & 5,76 & 5,68 & 5,65 \\
\hline 1,94 & 6,21 & 6,13 & 6,07 \\
\hline 3,88 & 8,15 & 8,09 & 7,98 \\
\hline 7,76 & 11,75 & 11,69 & 11,97 \\
\hline 8,54 & 12,47 & 12,69 & 12,69 \\
\hline 11,64 & 15,85 & 15,90 & 15,52 \\
\hline
\end{tabular}

Tabelle 3

Vergleich der Bestimmung des Ammoniak-Gehaltes im Meerwasser von der Helgoland-Reede nach der NaOBr-Bordeauxrot- und der Dichlorcyanurat-Methode. Die mit ** bezeichneten Proben waren tiefgefroren, die restlichen Proben wurden unfiltriert gemessen

\begin{tabular}{|c|c|c|}
\hline Datum & $\begin{array}{l}\text { Gefunden mit der } \\
\mathrm{NaOBr-Bordeauxrot-Methode} \\
\text { in } \mu \mathrm{g} \text {-at } \mathrm{NH}_{4}^{+}-\mathrm{N} / 1\end{array}$ & $\begin{array}{c}\text { Gefunden mit der } \\
\text { Didhlorcyanurat-Methode } \\
\text { (Autoanalyzer) in } \\
\mu \text { g-at } \mathrm{NH}_{4}^{+}-\mathrm{N} / 1\end{array}$ \\
\hline $19.11 .1971 \%$ & 3,34 & 3,72 \\
\hline $24.11 .1971 *$ & 3,50 & 3,47 \\
\hline $26.11 .1971 \%$ & 3,06 & 3,00 \\
\hline 1. $12.1971^{2}$ & 4,73 & 4,75 \\
\hline 3. $12.1971 \%$ & 1,72 & 1,71 \\
\hline 6.12.1971 & 3,78 & 3,75 \\
\hline 13. 12.1971\% & 3,95 & 4,42 \\
\hline 15. 12. $1971^{*}$ & 2,67 & 2,67 \\
\hline 17.12.1971 & 4,78 & 4,74 \\
\hline $22.12 .1971 \%$ & 3,72 & 3,70 \\
\hline 11. 2.1972 & 5,84 & 5,82 \\
\hline 14. 2.1972 & 5,56 & 5,54 \\
\hline 21. 2.1972 & 4,28 & 4,26 \\
\hline 25. 2. 1972 & 11,45 & 11,47 \\
\hline 29. 2.1972 & 5,12 & 5,10 \\
\hline 6. 3.1972 & 2,62 & 2,60 \\
\hline 8. 3.1972 & 4,17 & 4,15 \\
\hline 10. 3.1972 & 7,34 & 7,28 \\
\hline 13. 3.1972 & 5,85 & 5,87 \\
\hline
\end{tabular}


sehen, daß die mit der Dichlorcyanurat-Methode gefundenen Werte etwas unter denen der $\mathrm{NaOBr}$-Bordeauxrot-Methode lagen. Unter Umständen ist das auf die geringere Empfindlichkeit der Dichlorcyanurat-Methode gegenüber den im Meerwasser anwesenden Aminosäuren zurückzuführen. Die geringe Zahl der Vergleichsbestimmungen erlaubt allerdings in dieser Richtung noch keine eindeutige Aussage.

\section{ZUSAMMENFASSUNG}

1. Es wird eine Methode zur Bestimmung von Ammoniak im Meerwasser beschrieben, die statt des Oxidationsmittelverbrauchs eine direkte Farbstoffbildung mit dem Ammoniak colorimetrisch zu messen erlaubt.

2. Die Methode beruht auf der Bildung eines chinoiden blauen Farbstoffs mit NatriumSalicylat als phenolischer Komponente und Natrium-Dichlorcyanurat als Halogenträger. Die meisten im Meerwasser anwesenden Substanzen stören die Reaktion nicht und werden auch nicht erfaßt. Die Methode ist vom Salzgehalt in weiten Bereichen (ca. 25 bis $40 \%$ ) unabhängig.

3. Da nur eine veränderliche Lösung gegen eine konstante Gegenlösung photometriert wird, läßt sich die Messung auch gut mit einem Autoanalyzer durchführen. Dazu wurde die Vorbereitung der Reagenzien für die Messung auf See stark vereinfacht.

4. Während bei der manuellen Methode streng auf eine lineare Abhängigkeit zwischen Konzentration und Extinktion geachtet werden muß, kann bei der automatischen Bestimmung die Ammoniak-Konzentration direkt durch die Peakhöhe ausgedrückt werden. Diese Unabhängigkeit von der Linearität gibt die Möglichkeit einer genaueren Bestimmung des Ammoniaks im Bereich geringerer Konzentrationen.

5. Streuung und Reproduzierbarkeit liegen bei ca. $\pm 3 \%$ im untersuchten Meßbereich $z$ wischen 0,35 und $16,60 \mu \mathrm{g}$-at $\mathrm{NH}_{4}^{+}-\mathrm{N} / \mathrm{l}$. Wichtig für die Bestimmung ist die genaue Einhaltung der Temperatur und Reaktionszeit; dies ist bei der Analyse mit dem Autoanalyzer ohne Schwierigkeiten möglich.

Danksagungen. Wir danken den Herrn Drs. W. HickeL und D. Siebers (Biologische Anstalt Helgoland), Dr. M. GIllbricht (Institut für Hydrobiologie und Fischereiwissenschaft der Universität Hamburg) und Dr. M. ErrhardT (Institut für Meereskunde an der Universität Kiel) für Kritik und Beratung bei der Abfassung des Manuskriptes. Frl. S. Onusen danken wir für die Mithilfe bei der Durchführung der Ammoniak-Bestimmungen.

\section{ZITIERTE LITERATUR}

Bohling, H., 1970. Untersuchungen über freie gelöste Aminosäuren im Meerwasser. Mar. Biol. 6, $213-225$.

GrLibricrt, M., 1961. Eine Methode zum direkten Nadhweis von Ammoniak in Seewasser. Helgolander wiss. Meeresunters. 8, 58-67.

HEAD, P. C., 1971. An automated phenolhypochlorite method for the determination of ammonia in sea water. Deep Sea Res. 18, 531-532.

KorolefF, F., 1970. Direct determination of ammonia in natural waters as indophenol-blue. Inf. Techqs Meth. Sea Water Analysis 3, 19-22. 
Ludbochinsky, B. \& Zalta, J.P., 1954. Microdosage colorimetrique de l'azote ammoniacal. Bull. Soc. Chim. Biol. 36, 1363-1366.

Marteli, A. E. \& Calvin, M., 1958. Die Chemie der Metallchelatverbindungen. Verl. Chemie, Weinheim, $576 \mathrm{~S}$.

Reardon, J., Foreman, J. A. \& Searcy, R. C., 1966. New reactants for the colorimetric determination of ammonia. Clinica chim. Acta 14, 404-405.

Richards, F. A. \& Kletsch, R. A., 1964. Tech. Rep. Dep. Oceanogr. Univ. Wash. 97, 1-17.

SAGI, T., 1966. Determination of ammonia in sea water by the indophenol method and ist application to coastal and off-shore waters. Oceanogr. Mag. 18, 43-51.

Solorzano, L., 1969. Determination of ammonia in natural waters by the phenolhypochlorite method. Limnol. Oceanogr. 14,799-801.

Strickland, J. D. H. \& Parsons, T. R., 1965. A manual of sea water analysis. Bull. Fish. Res. Bd Can. 167, 311.

WAGNER, R., 1971. Vorläufige Arbeitsvorschrift. Bestimmung von Ammoniak bzw. Ammoniumionen in Wasser. Inst. für Siedlungswasserbau und Wassergütewirtschaft der TH Stuttgart, $2 \mathrm{pp}$.

Anschrift des erstgenannten Autors: R. BENESCH

Biologische Anstalt Helgoland

(Meeresstation)

2192 Helgoland

Bundesrepublik Deutschland 